\title{
Mit japanischer Faser
}

\section{Die Sicherung einer tintenfraßgeschädigten Choralhandschrift}

Die Handschrift auf dem Tisch der Restaurierungswerkstatt ist 475 Jahre alt. Der helle Schweinsledereinband hat Kratzer, Flecken und Fehlstellen. Beim Öffnen des Chorbuches zeigen sich ebenfalls Fehlstellen, Risse und beschädigte Bereiche im beschriebenen Papier, die braun-schwarze Tinte ist durchgeschlagen und wird von einem charakteristischen Geruch begleitet: die Handschrift hat einen Tintenfraßschaden.

Die Choralhandschriften der Württembergischen Landesbibliothek (Cod. mus. I) sind zumeist großformatige und schwere Folianten, deren Einbände mit Leder bezogen und durch feine Blindprägungen verziert wurden. Für die Textzeilen und die vorherrschend quadratischen Notationen, die Neumen, verwendeten die Schreiber meist schwarze Eisengallustinte, für Hervorhebungen rote Tinte. Aus dem Repertoire der Stuttgarter Hofkapelle stammen Notenhandschriften auf Hadernpapier, die einzigartige Einblicke in Repertoire und Aufführungspraxis einer überregional einflussreichen Musikinstitution des 16. Jahrhunderts gewähren. Diese Sammlung besitzt deutschlandweit nur in den Beständen der Bayerischen Staatsbibliothek München und der Universitätsbibliothek Jena vergleichbare Gegenstücke. Zum anderen stammen die Chorbücher aus verschiedenen Klosterbeständen, deren älteste Choralhandschriften im späten 12. bis 14 . Jahrhundert auf Pergament geschrieben wurden. Sie gelangten größtenteils 1803 mit der Überführung des Säkularisationsbestandes in die Bibliothek.

Die großformatigen Musikhandschriften dienten ursprünglich als Vorlage für den Chorgesang. Dafür wurde das Buch geöffnet und auf ein hohes Pult gelegt, vor dem sich die Sänger im Halbrund aufstellten, sodass die Seiten und großen Noten für alle einsehbar waren. Knicke, Risse und Griffkanten am Buchblock sind neben aufgespaltenen Deckelkanten, Kratzern und Fehlstellen im Lederbezug der Einbände klassische Gebrauchsspuren, die uns noch heute die Nutzung der Bände verdeutlichen.

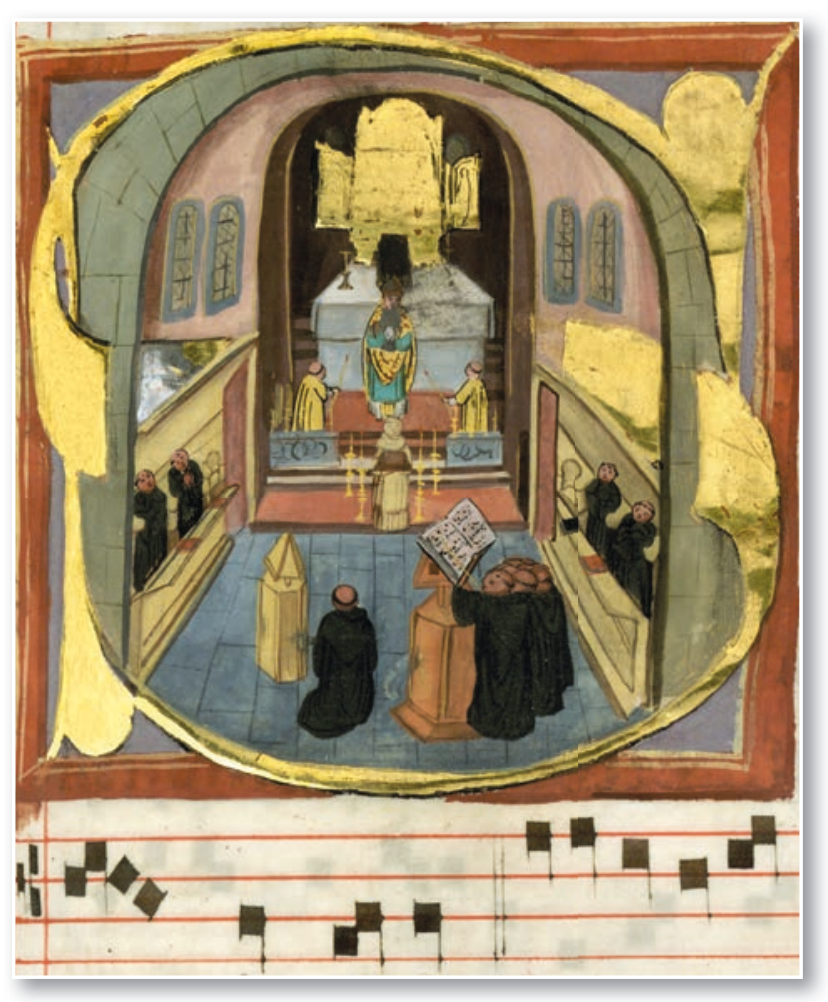

Abb. 1: Bildinitiale mit der Darstellung eines Chorbuchs während eines klösterlichen Gottesdienstes (Cod. mus. I 2 63, Blatt 151 recto)

\section{Tintenfraß}

Für den besonders schlechten Erhaltungszustand mehrerer Chorbücher ist jedoch ein anderer Grund ausschlaggebend: die verwendete Eisengallustinte. Sie wird seit dem 3. Jahrhundert $\mathrm{n}$. Chr. als historisches Schreibmittel hergestellt. Schon beim Herstellungsprozess wird der Grundstein für später auftretende Schäden gelegt:

Eisengallustinten basieren auf einem wässrigen Sud aus Galläpfeln (Gallsäurehaltige, kugelförmige Auswüchse an Eichenblättern, die durch die Gallwespe beim Einlagern ihrer Eier entstehen) und Eisen- oder Kupfervitriol' ${ }^{1}$, dem Gummi arabicum beigemengt wurde. ${ }^{2}$

Beim Auftragen ist die frisch hergestellte schwarzbläuliche Tinte noch sehr hell. Zusammen mit dem Luftsauerstoff färbt sie sich je nach Zusammensetzung hellbraun bis schwarz und bildet eine wasserfeste, nicht löschbare Beschriftung auf Papier oder Pergament, die als dokumentenecht bezeichnet wird.

1) Eisen- und Kupfervitriol sind Salze, die als Mineralien im Bergbau oder bei der Alaunherstellung gewonnen wurden.

2) The Iron Gall Ink Website; https://irongallink.org/igi_indexee73.htm/ 
Durch die im Vitriol enthaltenen, natürlich schwankenden Anteile von Eisen(II)sulfaten können in manchen Tinten überschüssige Eisenionen nicht mit ausreichend Gallsäure zum Tintenfarbstoff reagieren und bleiben ungebunden. Außerdem entsteht bei der historischen Tintenherstellung ein unterschiedlicher Anteil an Schwefelsäure. Unter dem Einfluss von schwankender Feuchtigkeit und Luftschadstoffen zersetzen diese löslichen, hoch reaktiven Tintenanteile die Papierfasern des Schriftträgers. Zu Beginn des Schadens sind die Ränder der Tinte ein wenig verschwommen und es entsteht ein bräunlicher Hof um das Schriftbild. Dann verstärkt sich die Verbräunung des Papiers bis auf die Blattrückseite. In dem zunehmend versprödeten Papier bilden sich schließlich kleinste Haarrisse bis hin zu ganzen Ausbrüchen beschriebener Bereiche.

Handschriften auf Papier, die fortgeschrittenen Tintenfraß aufweisen, sind im Schriftbereich nicht mehr flexibel beweglich. Schon allein die Bewegung des Blätterns lässt Risse entstehen und die Schrift aus dem abgebauten Papierverbund herausbrechen. Diese Schäden sind irreversibel und der Zerfallsprozess kann sich bei schlechten Lagerungsbedingungen und besonders durch hohe Luftfeuchte zusätzlich noch beschleunigen.

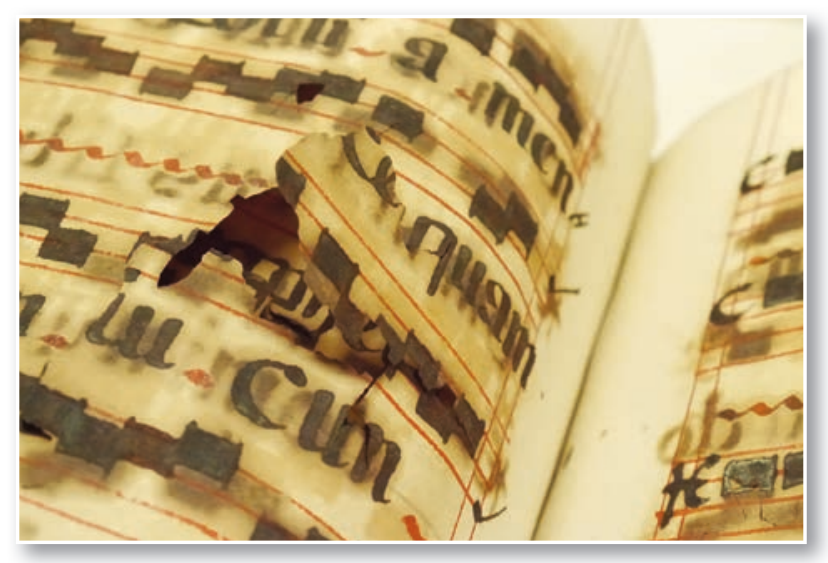

Abb. 2: Fortgeschrittener Tintenfraßschaden

Viele Bände der Stuttgarter Chorbücher können heute aufgrund dieser Schäden nur in Ausnahmefällen im Original eingesehen werden. Mehrere Handschriften dieser Sammlungsgruppe sind inzwischen sogar von der Benutzung ausgeschlossen, da sich die im zersetzten Papier bestehenden Risse und Ausbrüche bereits durch das Blättern vermehren. Eine Schutzverfilmung und Digitalisierung, wie sie

3) Wichmann, Natascha: Neue Ansätze zur Anwendung von remoistenable tissues für die Sicherung von fragmentierten Eisengallustinten-Dokumenten [Bachelorarbeit]. Staatliche Akademie der Bildenden Künste. Stuttgart 2017. am Bestand herausragender Handschriften in der WLB durchgeführt wird, ist deshalb aufgrund der mechanischen Beanspruchung bisher nicht ohne markante Informationsverluste möglich.

Behandlungsmethoden zielen darauf ab, die langsam fortschreitende Zersetzung des Papiers nachhaltig zu verhindern und eine Festigung geschädigter Bereiche zu erreichen. Diese Verfahren können aktuell nur an Einzelblättern im Bad angewendet werden oder mit dem Fokus auf eine lokale Papierstabilisierung. Sie werden ständig weiter entwickelt und optimiert, und ihre Langzeitwirkung von Konservierungswissenschaftlern geprüft.

Die betroffenen tintenfraßgeschädigten und zugleich original in Bindung und Einband erhaltenen

Stuttgarter Chorbücher stellen aktuell für die Restaurierung eine Schwierigkeit dar: Die fragile Beschaffenheit der Seiten und der gebundene Zustand schließen eine wässrige Behandlung aus.

Ziel einer Behandlung ist daher die Festigung der Schriftbereiche, die eine anschließende Digitalisierung durch geschultes Personal im Haus möglich macht.

\section{So wenig wie möglich - so viel wie nötig: die Sicherung der Choralhandschrift Cod. mus. I fol. 32}

Im aktuellen Zustand kann für die betroffenen Chorbücher eine für die Digitalisierung ausreichende Stabilisierung fragiler Bereiche mit sehr dünnem Japanpapier erreicht werden, das mit Gelatine als Klebstoff beschichtet ist und in kleinen Streifen, sogenannten Brücken, über Risse und kleinere Fehlstellen verklebt wird. Ausgebrochene Fragmente können auf diese Weise wieder an originaler Stelle eingesetzt werden.

Dieser Behandlungsansatz wurde im Zuge einer Kooperation mit der Staatlichen Akademie der Bildenden Künste in Stuttgart an der ersten Handschrift der Sammlung umgesetzt. In enger Abstimmung mit der Restauratorin der WLB hat Natascha Wichmann vom Studiengang "Konservierung und Restaurierung von Kunstwerken auf Papier, Archivund Bibliotheksgut" wissenschaftliche Voruntersuchungen und die nachfolgenden Sicherungsmaßnahmen durchgeführt. ${ }^{3}$

Zunächst wurden an Testpapieren mit simulierten Tintenfraßschäden verschiedene Japanpapierstärken 
mit Klebstoffbeschichtung und deren Festigungseigenschaften verglichen. Eine große Rolle spielten dabei auch die Intensität und Art der Befeuchtung und Handhabung des beschichteten dünnen $\mathrm{Pa}$ piers.

In der Restaurierungswerkstatt der Württembergischen Landesbibliothek wurde nach Abschluss und Bewertung der Vorarbeiten im Juli 2017 mit den praktischen Sicherungsmaßnahmen begonnen. Als erstes wurde der vorliegende Zustand dokumentiert und fotografiert. Durch eine behutsame Trockenreinigung konnte der Oberflächenschmutz entfernt werden. Fragmente, die sich im Falzbereich des Buches und über die folgenden Seiten verteilt hatten, wurden gesichert.

Zur Stabilisierung der Papierschäden am ausgewählten Band wurde der Einsatz eines Japanpapiers mit einem Flächengewicht von 1,6 g/ $\mathrm{m}^{2}$ gewählt4. Dieses dünne Papier unterstützt die Bewegung des Blattes optimal, ohne selbst einzureißen. Es lässt sich trotz der geringen Grammatur beim manuellen Verkleben gut verarbeiten. Da dieses Japanpapier nicht mit einem Gelatinefilm beschichtet gekauft werden kann, muss der spezielle Klebstofffilm selbst aufgebracht werden. Ein direktes Bestreichen mit der dünnflüssigen Gelatine würde das zarte Papier jedoch einreißen lassen und beim Anbringen zu viel unkontrollierte Feuchte in das beschriebene Blatt leiten. Daher wird das dünne Papier in einen, auf einer Silikonunterlage ausgestrichenen, frischen Gelatinefilm gelegt.

Nachdem die Gelatine zu einem festen Klebstofffilm getrocknet ist, lässt sich das nun beschichtete Japanpapier in kleine Streifen, sogenannte Brücken, zuschneiden.

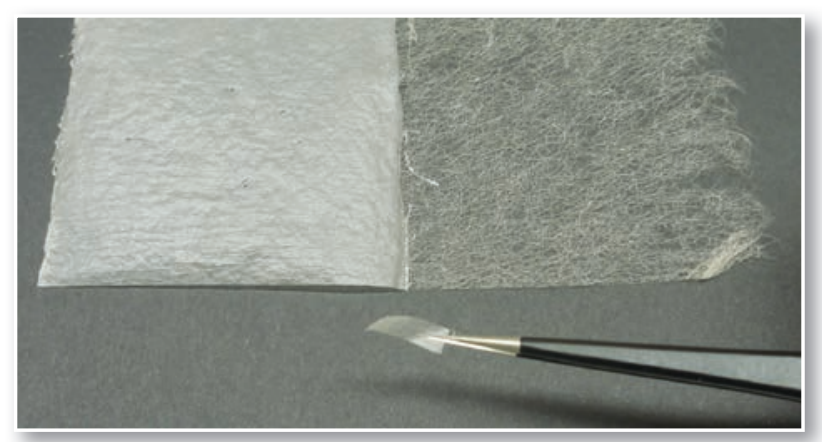

Abb. 3: Japanpapier mit Klebstofffilm (linke Hälfte) und ein zugeschnittener Streifen
Zunächst werden alle Bereiche auf der Vorderseite bearbeitet: Durch das fein dosierte Benetzen mit einem Wasserpinsel wird der Gelatinefilm angefeuchtet und das beschichtete Japanpapier kann nun mit streichenden Pinselbewegungen angedrückt und verklebt werden. Anschließend müssen die bearbeiteten Stellen vollständig trocknen. Erst dann kann die Rückseite zur beidseitigen Sicherung der Schäden behandelt werden.

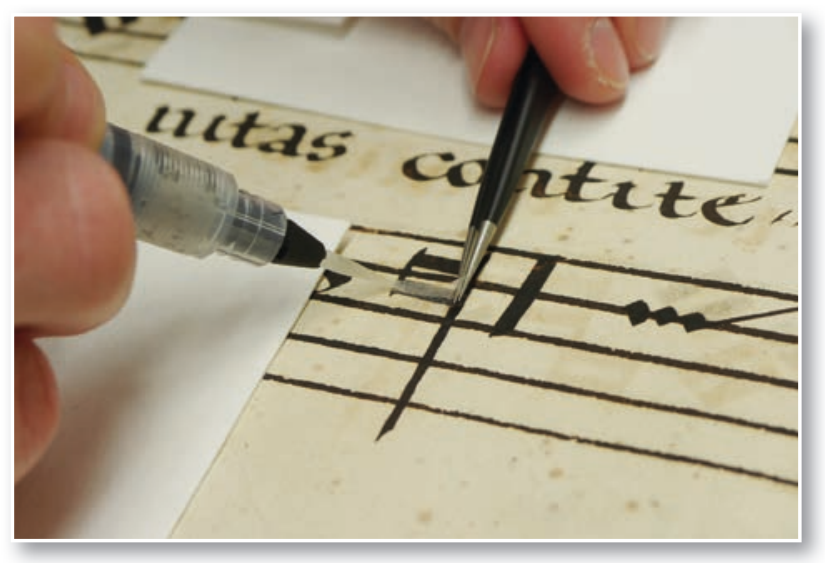

Abb. 4: Verkleben einer Brücke aus Japanpapier

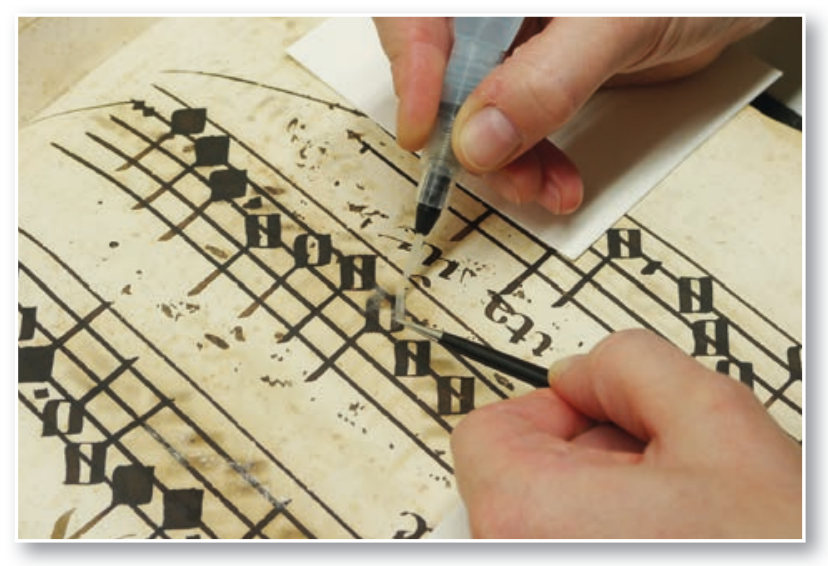

Abb. 5: Stabilisierter Bereich (unten links im Bild)

Der Tintenauftrag der Handschrift Cod. mus. I fol. 32 stellte eine weitere Schwierigkeit dar. Die Tinte wurde ungleichmäßig dick aufgetragen, da der Schreiber eine natürliche Schreibfeder verwendete, die ungleiche Mengen an Flüssigkeit abgab. Das Papier ist unter intensiven Tintenaufträgen lokal stärker geschädigt. An den betreffenden Stellen im Schriftbild zeigten sich nicht nur kleine Haarrisse und Ausbrüche, die Tinte pulvert dort außerdem vielerorts ab. Die Tintenpartikel lösen sich durch das Aufstreichen des Japanpapieres mit dem Pinsel ab und können sich im umgebenden Papier ablagern. Es war erforderlich, diese Bewegung so weit wie möglich zu reduzieren. Durch das Auflegen auf ein 
feuchtes Schwammtuch kann das Japanpapier kontrolliert benetzt und anschließend mit einer Pinzette und Pinsel auf die vorbereitete Stelle im Schriftbild der Handschrift positioniert und angedrückt werden.

Jegliche Zugluft gilt es zu vermeiden, denn das aufgewirbelte, dünne Japanpapier wird unkontrolliert ineinander gefaltet oder ganz vom Arbeitstisch geweht. Eine Lupenlampe erleichtert zusätzlich die Arbeiten: Durch ein reflektierendes Streiflicht wird z. B. überschüssige Feuchtigkeit sofort sichtbar und kann abgenommen werden. Herausgebrochene Fragmente können unter der Vergrößerung passgenau positioniert werden.
Die Handschrift konnte mit dieser Methode erfolgreich in-situ und punktuell an allen geschädigten Stellen bearbeitet werden. Die Trockenreinigung der fragilen Handschrift erforderte einen Zeitaufwand von 12 Stunden.

Aufgrund des Schadensbildes für die lokale Sicherung der tintenfraßgeschädigten Schriftbereiche von fol. 40 bis 122 ergab sich ein mehrtägiger Arbeitseinsatz von insgesamt 32 Stunden.

Die nun für den Bestand erprobte Arbeitsmethode kann und soll künftig an weiteren Chorbüchern mit Tintenfraßschäden angewendet werden, um die zuvor nicht benutzbaren Bücher für eine Digitalisierung ausreichend zu stabilisieren und auf diese Weise wieder für die Forschung zugänglich zu machen.

Sonja Brandt

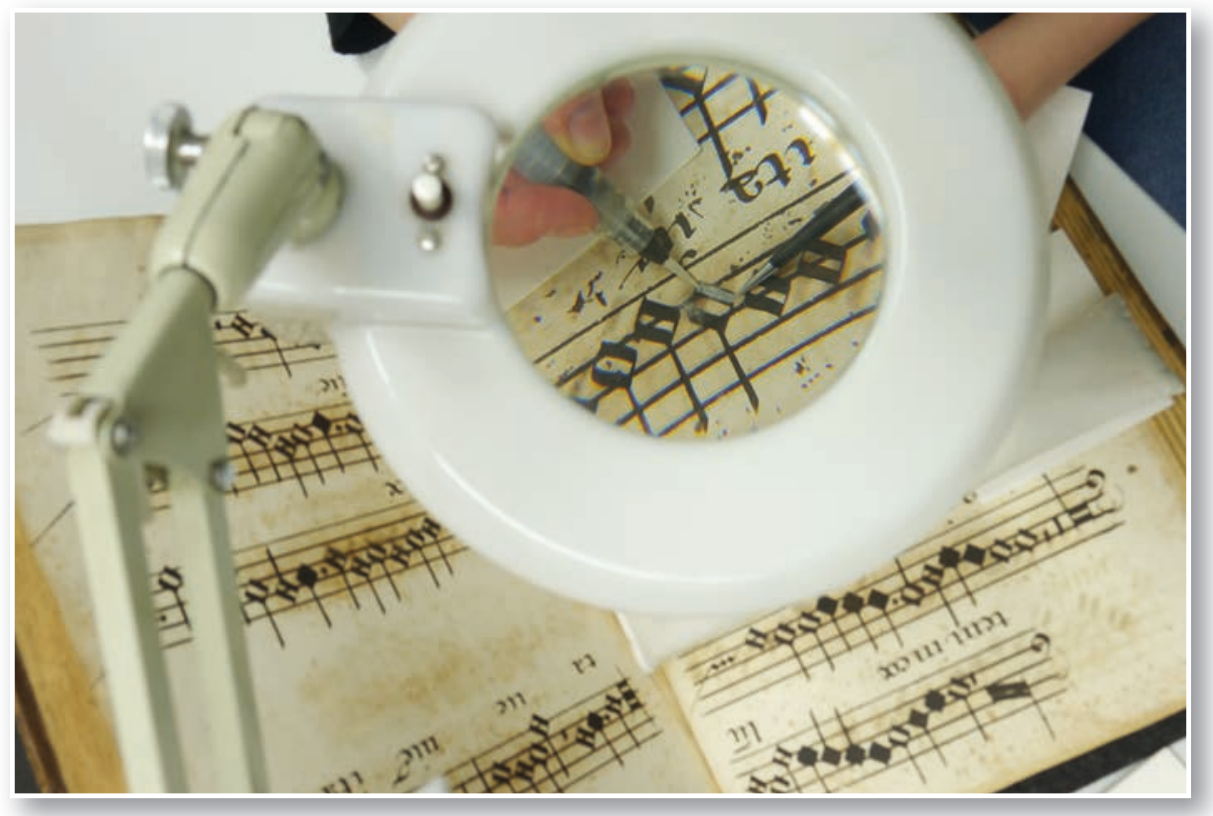

Abb. 6: Arbeit unter der Lupenlampe

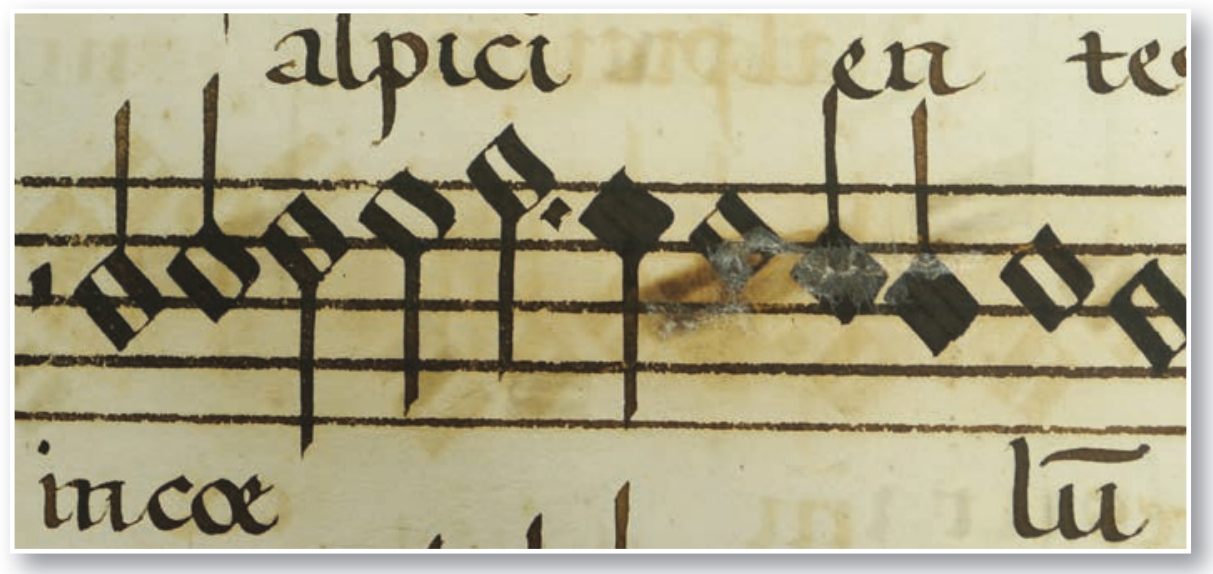

Abb. 7: Stabilisierter Bereich 